Meas. Sci. Technol.

\title{
A Tunable supercontinuum laser using a Digital Micromirror Device
}

\section{Tobias C. Wood, Daniel S. Elson}

Hamlyn Centre for Robotic Surgery, Institute of Global Health Innovation and Department of Surgery and Cancer, Imperial College London

\begin{abstract}
Spectral filtration systems based around Digital Micromirror Devices (DMD) have made possible the creation of illumination light with custom spectral profiles for microscopy and endoscopy. However due to the out-of-plane deviation caused by the DMD these systems have previously required complex optics, and in addition the diffraction effects of the DMD have not been well understood. We present a system that incorporates a Total-Internal Reflection (TIR) prism to significantly simplify the optics and also a full two-dimensional analysis of the diffraction patterns from the DMD. Full spectral coverage between 430 and $720 \mathrm{~nm}$ was demonstrated with the output coupled into a liquid light guide for easy coupling into surgical devices such as laparoscopes.
\end{abstract}




\section{Introduction}

Light sources for biomedical optics applications can be roughly divided into two types; white-light sources for general purpose illumination, which can be optionally shaped by spectral filtering for specific applications, and narrow-band laser sources. These laser sources are often limited to a selection of wavelengths that coincide with specific laser transitions and can limit their use in applications such as fluorescence or multispectral imaging, where a laser source needs to be matched to a particular fluorophore or absorption feature. The use of filtered white light lamp sources can achieve some flexibility in illumination wavelength, but the large number of filters required for highly multispectral or hyperspectral imaging still imposes a practical limit on the spectra available for a particular experiment. For the thorough study of multispectral signals a flexible light source that could be tuned to a particular wavelength, bandwidth or custom excitation spectrum would be useful. We have achieved broadband and arbitrary tunability of a supercontinuum source through the modelling and theoretical analysis of the diffraction, interference and linearity aspects of the light-Digital Micro-mirror Device (DMD) interaction.

Digital Micro-mirror Device (DMD) devices form the core of Digital Light Processing technologies, which spatially modulate light in order to project an image. This technology has also been used in applications ranging from laser beam shaping [1], to 3D imaging with structured light [2]. We instead use the DMD in combination with a dispersive element to spectrally filter a white-light supercontinuum source. This allows us to not only tune to particular wavelengths of interest but also to select a custom spectral profile.

Previous work has shown the feasibility of using spatial modulation to selectively filter a white-light source. The general scheme of such systems is to laterally separate wavelengths using a dispersive element such as a prism, and then selectively transmit or reflect desired wavelengths. Dunsby et al. demonstrated such a scheme using a supercontinuum source, prism, and a motorised slit to pass a single band of wavelengths $[3,4]$.

Commercial devices using a Xenon arc lamp and DMD as the filtration device are now available (OneLight Spectra, OneLight Corp. and OL-490 Agile Light Source, Optronic Laboratories), and these sources have been used for surgical and medical applications, but suffer from reduced efficiency or reduced spectral resolution due to the compromises in focusing through a slit, as well as requiring complicated optics such as an anamorphic lens to collect the light from the DMD [5, 6, 7]. Other groups have detailed the use of a supercontinuum as the light source, but these also required complex optics or had limited spectral resolution in the visible range [8, 9].

One of the limitations of the use of the supercontinuum in this application is the diffraction and interference that occurs due to the structure and operation method of the DMD device. We present here a detailed discussion of the diffraction effects

of the DMD and also a method to linearize the system response. A device that 
incorporates a low-cost Total Internal Reflection (TIR) prism has been modelled and built, which significantly reduces the complexity of the required optics for the system. The instrument has potential applications in microscopy and endoscopy for multispectral excitation or illumination with custom spectra.

\section{System design and construction}

Although the most common high power white light source in general medical use is the Xenon arc lamp and high illumination powers of over $100 \mathrm{~W}$ are available, the output of such lamps is poorly collimated and difficult to condense to a small spot. These features result in an inefficient light transmission through more complex optical systems such as monochromators or optical fibres.

An alternative white light source that has been developed and commercialised in recent years is the supercontinuum laser. The formation of a supercontinuum is highly complex and involves several different non-linear optical processes [10]. In the commercially available supercontinuum used in this study, picosecond pulses of highly mono-chromatic laser light are injected into the anomalous group velocity dispersion regime of a highly non-linear PCF, where soliton break-up and four-wave mixing processes produce a massively broadened supercontinuum spectrum. The supercontinuum used in this study has a quoted total optical power of 6 Watts integrated over the wavelength range 400-1700 nm (Fianium Ltd., UK, model SC-400-6).

A DMD consists of a rectangular array of square aluminium mirrors attached to the surface of a semiconductor chip [11, 12]. Each of these mirrors is microscopic and mounted on a torsion hinge that runs diagonally between two opposite corners. We used a 0.7" XGA format DMD (Texas Instruments Ltd) mounted in a Discovery 1100 controller board (Vialux $\mathrm{GmbH}$ ), with a mirror edge length of $13.68 \mu \mathrm{m}$. Several previous groups have used a DMD to produce custom spectra, by focussing a dispersed spectrum onto a DMD and then collecting the reflected light $[5,8,9,13,14]$. However the mirror tilt direction causes significant practical issues during system design, as a ray at normal incidence to the plane of the DMD is reflected through an angle of twice the mirror tilt out of the plane. The DMD we used has a mirror tilt angle of only 12 degrees, and the resulting 24 degree out of plane deviation of rays means that subsequent optics must be mounted at a significant distance to the DMD to ensure sufficient path separation.

There have been several different solutions to the mirror tilt issue in the literature. McConnell et al. [8] attempted to use a DMD with a supercontinuum but did not use a second prism and only produced three distinct spectral bands. Chuang and Lo designed a system that could produce arbitrary profiles using an erbium-doped fibre amplifier with a central wavelength of $1550 \mathrm{~nm}$ and bandwidth of $37 \mathrm{~nm}$ [13]. The narrow wavelength range used lessened the impact of diffraction effects described later in this paper. MacKinnon [5] and Brown et al. [14] used custom designed anamorphic collection optics to solve this problem, while more recently Rice et al. demonstrated a system that 


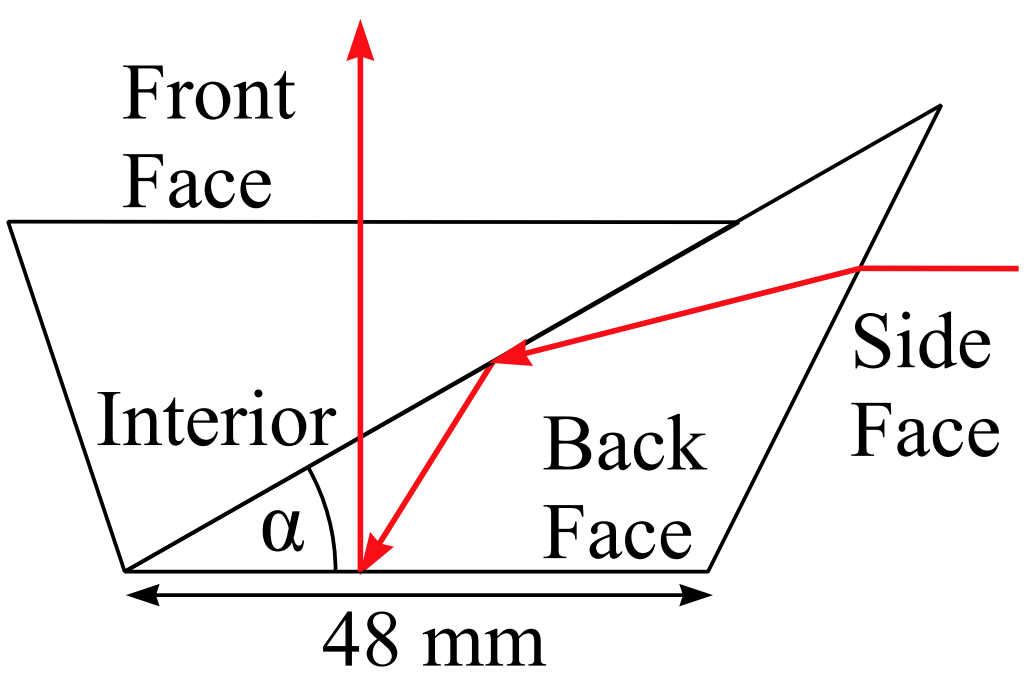

Figure 1. A Total Internal Reflection (TIR) prism showing how a ray propagates through the interfaces from the side to the front.

uses a second prism and an integrating sphere to collect sufficient light. [9, 15, 16].

To simplify the system design and avoid this problem we incorporated a TotalInternal Reflection (TIR) prism into the optical path (Ocean Films Ltd). A TIR prism consists of two angled prisms cemented together so that one pair of exterior faces are parallel and are widely used in commercial DLP (Digital Light Projection, trademark Texas Instruments) systems. As depicted in figure 1 these further fold the light path to increase the separation of the incident and reflected light from the DMD. Light incident on the front or back face at perpendicular incidence will pass through the thin layer of optical cement between the two prisms with zero angular deviation. However light incident on the side face will strike the interior surface above the critical angle and so will undergo total internal reflection and be directed toward the back face. The internal prism angle $\alpha$ and external angle $\beta$ are tuned to match the rotation angle of the DMD which is mounted immediately behind the back face. Rays are then reflected normal to the back and front face, and are hence below the critical angle of the internal face.

This folding of the light path produces a much greater separation of the incident and reflected light channels. Without the prism a large optical path would be required to physically separate illumination and collection optics given the small $24^{\circ}$ path deviation the mirrors introduce. With the prism, rays are deflected by approximately $90^{\circ}$ in total, allowing for a shorter optical path between components.

The system layout, including ray traces of wavelengths between 425 to $640 \mathrm{~nm}$, is given in figure 2. These traces were calculated using custom software to allow the ray paths to be visualised through the system. Figure 2 shows both a top-down plan view of the complete system and an orthogonally projected 3D view to illustrate the two horizontal mounting planes required by the use of the DMD and TIR prism. 


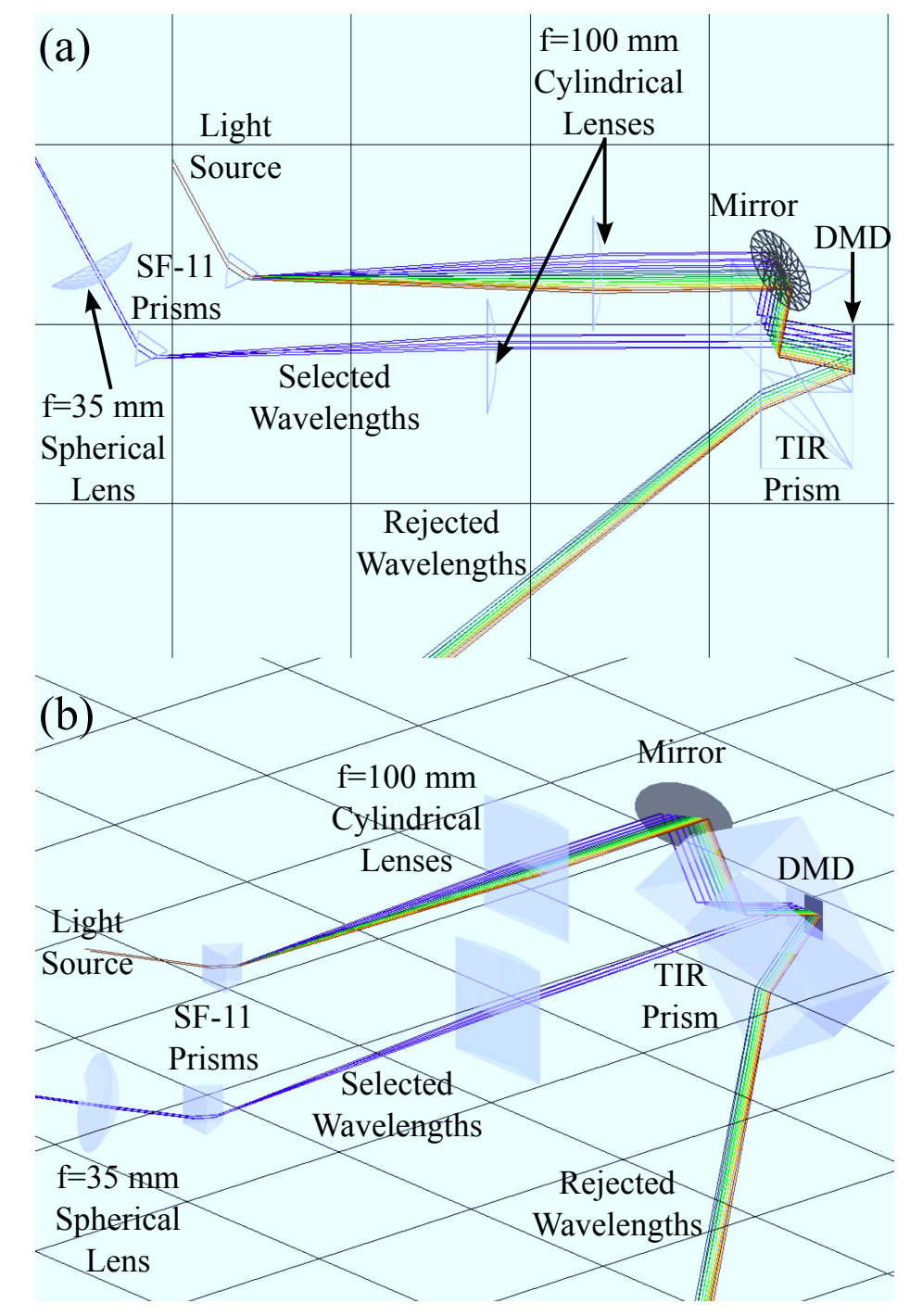

Figure 2. (a) Top down plan view and (b) orthogonally projected 3D view of the complete system. The rays between the light source and the mirror, and the TIR prism and the spherical lens are parallel to the depicted horizontal plane. The other ray paths depicted are out of the horizontal plane due to the orientation of the mirror, the TIR prism (oriented $45^{\circ}$ from horizontal) and the DMD mirrors (rotation axis oriented $45^{\circ}$ from horizontal).

The output of the supercontinuum was collimated with a lens and then dispersed using an SF-11 prism. The spectral envelope was consequently collimated and individual wavelengths focussed in the horizontal direction using a $100 \mathrm{~mm}$ focal length cylindrical lens. In figure 2 the rays between the light source and mirror are parallel to the depicted horizontal plane. The mirror then deflects the rays at 45 degrees to the horizontal, and they make a first pass through the TIR prism at this angle. The TIR prism was oriented with the long side at 45 degrees to the horizontal and placed in front of the DMD. The DMD was mounted with the long edge horizontal and the short edge vertical. The DMD micro-mirrors then reflect the rays back into the horizontal plane, where they take a 
different path through the TIR prism and continue to the cylindrical lens. The total path length between the cylindrical lens to the front of the DMD was $100 \mathrm{~mm}$.

In the absence of diffraction effects the light from the DMD could be re-collimated in the horizontal direction using a matching lens and prism to those used before the DMD. The second prism should be oriented the same as the first as the order of the colors in the spectrum is reversed with respect to the propagation direction after reflecting off the DMD. The two lenses must be the same focal length to produce incidence angles at the second prism matching the exit angles at the first prism. The final component in the system is a $35 \mathrm{~mm}$ focal length spherical lens to focus the output light for collection.

\section{System characterization}

\subsection{Diffraction Analysis}

The use of the DMD with a coherent light source gives rise to significant diffraction artefacts. Previous discussion of these has been centred on approximating the DMD as a one-dimensional blazed reflection grating as illustrated in figure 3. In an ordinary grating the grating and mirror normals are coincident and the majority of the power will be directed into the central diffraction order which has no wavelength dependency on angular deviation. The blaze angle shifts power into a higher diffraction order with a wavelength dependency, resulting in stronger separation of wavelengths by the grating [17].

(a)

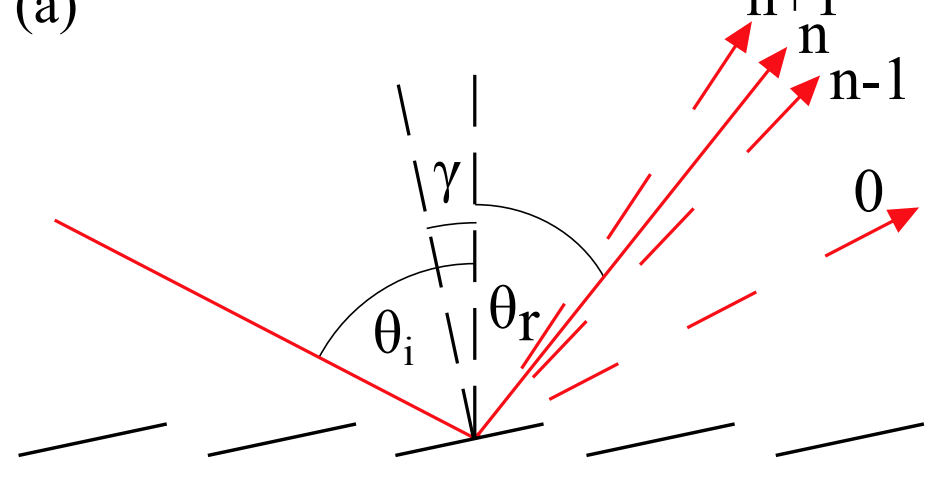

(b)

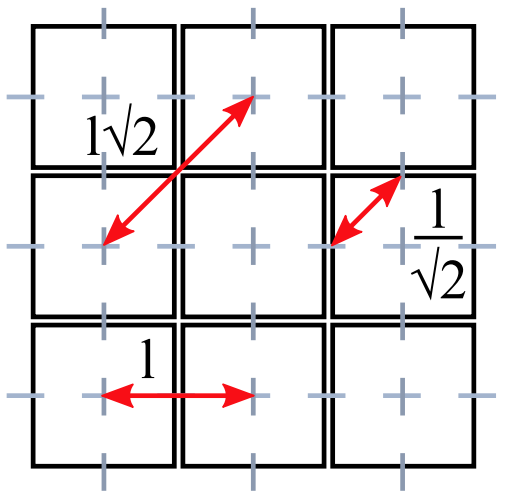

Figure 3. (a) A one dimensional blazed grating and (b) a schematic showing possible 'pitches' for different cross-sections of the DMD.

When applying this analysis to the DMD there is an ambiguity over what value to choose for $d$, the grating pitch and mirror size, as these are not constant in the direction perpendicular to the mirror tilt. As shown in figure 3 there are two possible cross-sections across the DMD that result in a constant pitch and element size, and the smaller value of $l / \operatorname{sqrt}(2)$ has been used in the literature. However other cross-sections in between these two will produce varying element sizes. This means that a simple 


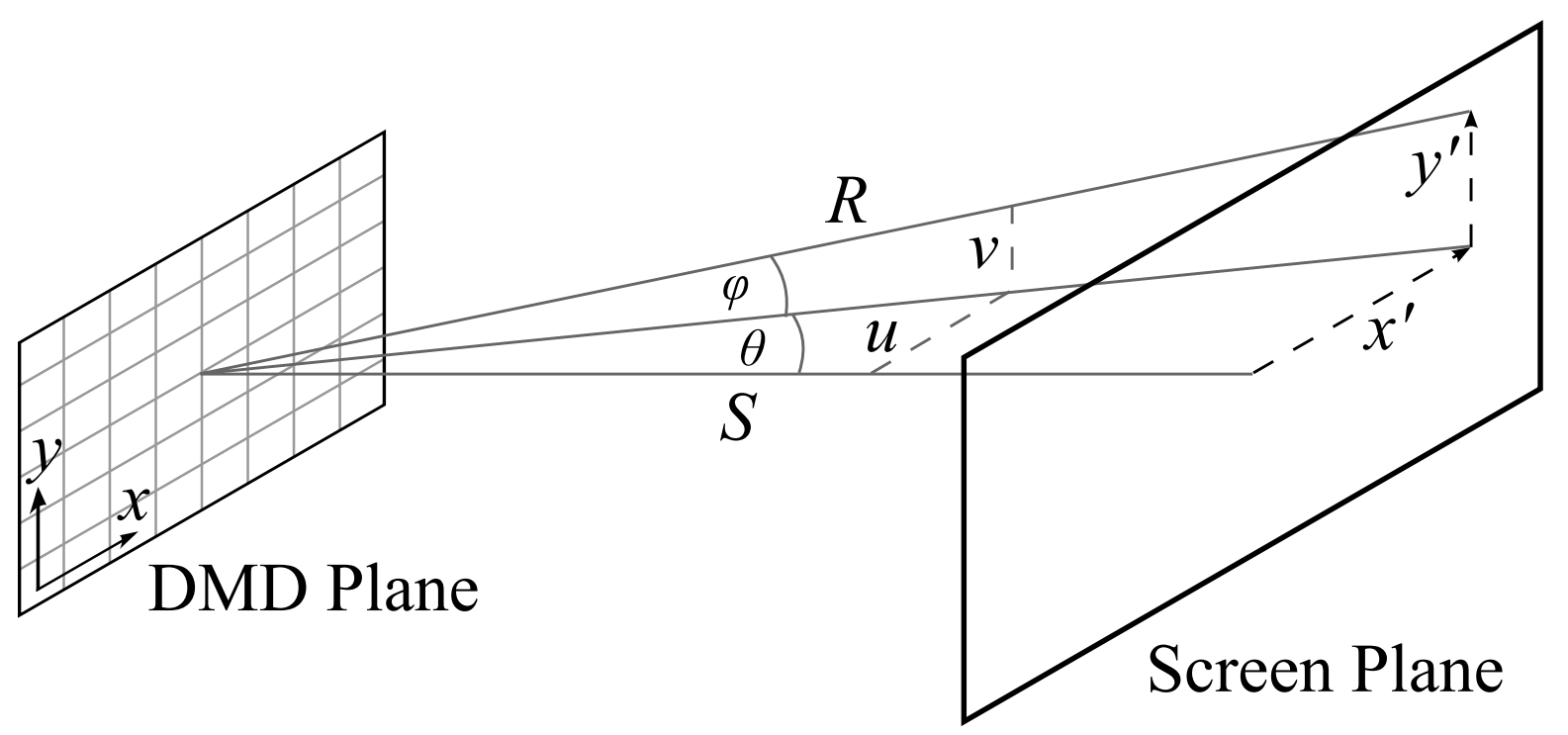

Figure 4. Co-ordinates used in each of the planes for the fourier analysis.

one-dimensional approximation of the DMD cannot adequately explain the observed diffraction pattern.

To fully understand the diffraction effects from the DMD a two-dimensional analysis is required [18]. A blazed grating can be modelled as a convolution of a triangular element function with a comb of Dirac delta functions, multiplied by an overall aperture or illumination function. We simulated this in two dimensions in order to find the farfield diffraction pattern that should be observed from the DMD. The arrangement and co-ordinate systems used are shown schematically in figure 4 . To convert between the generalised spatial frequency co-ordinates $(u, v)$ to physical locations on a screen $\left(x^{\prime}, y^{\prime}\right)$ at distance $S$ from the aperture the following relationship was used [17]:

$$
\begin{aligned}
& u=\frac{\sin \theta}{\lambda} \\
& u=\frac{x^{\prime}}{\lambda R \cos \phi} \\
& u \approx \frac{x^{\prime}}{\lambda S}(R \approx S, \cos \phi \approx 1) \\
& x^{\prime}=\lambda S u
\end{aligned}
$$

where $R$ is the distance from the aperture origin to the point $\left(x^{\prime}, y^{\prime}\right)$, which in the farfield can be approximated by $S$, and $\phi$ is shown in figure 4. A similar result applies for $y^{\prime}$.

To simulate the DMD aperture the height across an individual mirror surface perpendicular to the DMD plane was approximated as a two dimensional triangular function $h(x, y)=x+y$ over the full extent of the mirror pitch. This is an approximation as the mirror edges are slightly smaller than the pitch so that they do not collide when switching. In addition the projection of a tilted square is a diamond shape, leading to a 
further reduction in the effective mirror area when tilted. This will lead to a negligible broadening of the overall envelope in the far-field and can be ignored [17]. The mirror elements can then be modelled as having constant amplitude and a phase determined by

$$
\psi(x, y)=\frac{2 \pi(x+y)}{\lambda}
$$

This element function was convolved with a two-dimensional grid of delta functions and then multiplied by an aperture function. In the $x$-direction this consisted of a top-hat function with the width of two mirrors to approximate that in reality it is unlikely that all wavelengths can be focussed to a perfect stripe on the DMD. The width was chosen based on the spectral FWHM measured In the $y$-direction a Gaussian distribution represented the supercontinuum intensity profile. A Fast Fourier-Transform (FFT) was then used to find the far-field diffraction pattern (Matlab, the Mathworks Plc.).

The full 1024 by 768 grid of mirrors could not be simulated due to memory constraints, but it was found that 48 sample points were required in each direction for a single mirror element in order to avoid aliasing effects. Hence a much smaller 32 by 32 grid of mirrors was simulated for each wavelength individually. However the important features of the far-field are determined by the element function and element spacing which were adequately simulated even in this small region of the DMD. The phase component of the central section of the aperture is shown in figure 5. Mirrors that fell outside of the lit region of the DMD gave zero phase change as their amplitude was also zero.

To combine the diffraction simulations of multiple wavelengths required an extra step of displacing the horizontal co-ordinates so that they originate at the location that each wavelength strikes the DMD. The deviation angle $\delta$ of a ray traversing a prism is given by [19]

$$
\delta=\theta+\arcsin \left(\sin \alpha \sqrt{n^{2}-\sin ^{2} \theta}-\sin \theta \cos \alpha\right)-\alpha
$$

where $\theta$ is the incident angle of the ray on the prism, $\alpha$ is the prism wedge angle and $n$ is the refractive index of the prism. The only variable in this equation that depends on the wavelength $\lambda$ is the refractive index, as once the system has been set up the others are constant. The refractive index can be calculated using the Sellmeier Equation:

$$
n^{2}=1+\sum_{i} \frac{B_{i} \lambda^{2}}{\lambda^{2}-C_{i}}
$$

where $B_{i}$ and $C_{i}$ are the Sellmeier co-efficients which describe the locations and strengths of the resonant frequencies of the material. Figure 6 shows the non-linear deviation angle for the SF-11 glass prism used in our system.

Ideally we would invert equations 6 and 7 in order to find the wavelength that is deviated by a particular angle, and hence strikes the DMD at a particular position. Unfortunately the three-term Sellmeier equation is intractable to invert, hence a numerical scheme was used to simulate the bandwidth expected from different columns 


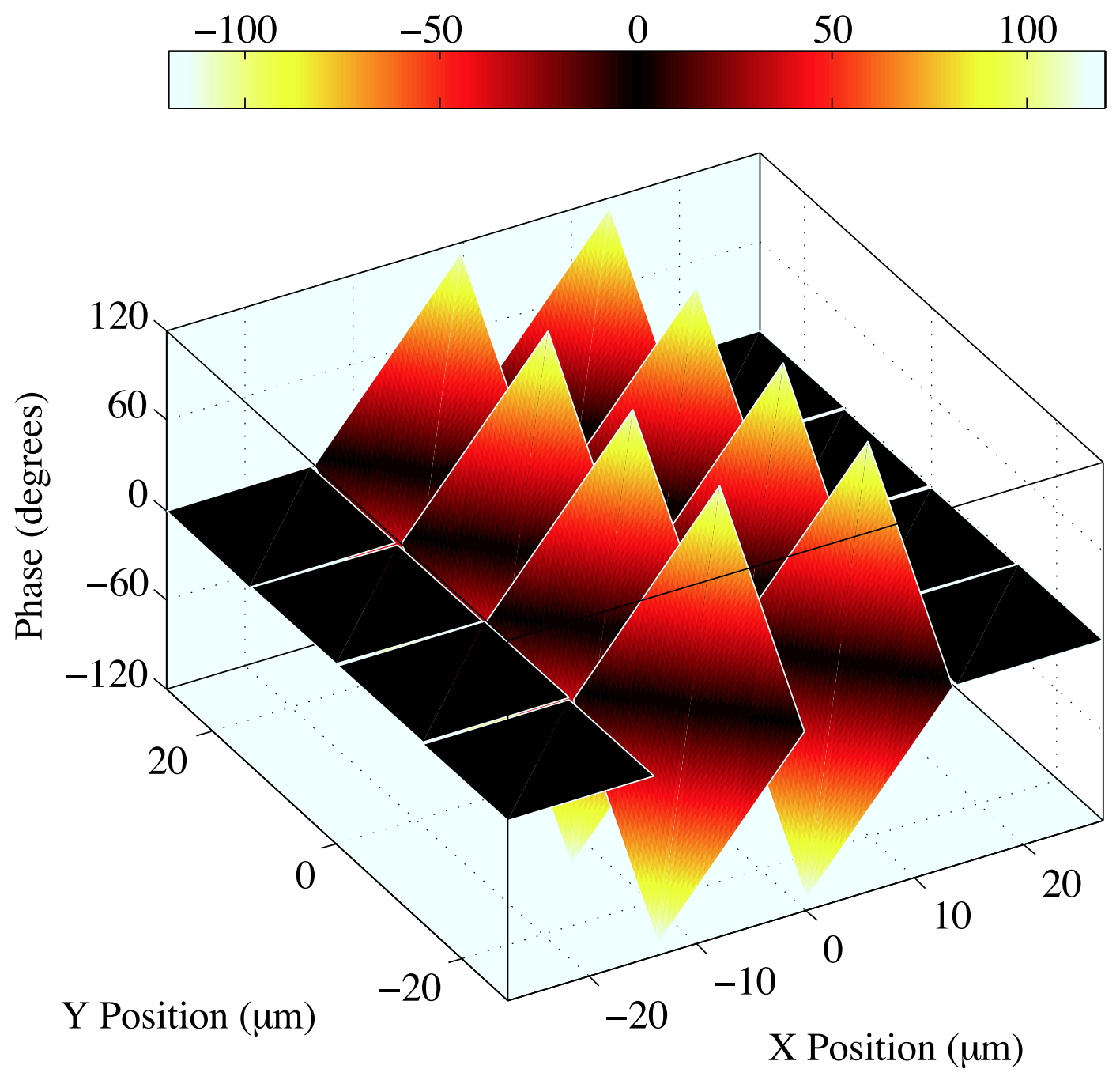

Figure 5. The simulated aperture phase function of the DMD.

of the DMD. Using the simplified optical system in figure 6, which neglects the pathfolding effects of the mirror and TIR prism, the position on the DMD of a particular wavelength is:

$$
\begin{aligned}
& x=f \sin \left(\delta_{m}-\delta\right) \\
& \delta_{m}=2 \theta-\alpha \\
& x=f \sin \left(\theta-\arcsin \left(\sin \alpha \sqrt{n^{2}-\sin ^{2} \theta}-\sin \theta \cos \alpha\right)\right)
\end{aligned}
$$

where $\delta_{m}$ is the minimum deviation angle [19], and $n$ is calculated using equation 7 . This assumes that the minimally deviated wavelength strikes the exact centre of the DMD, hence the position $x$ can extend up to half the DMD width in each direction. 

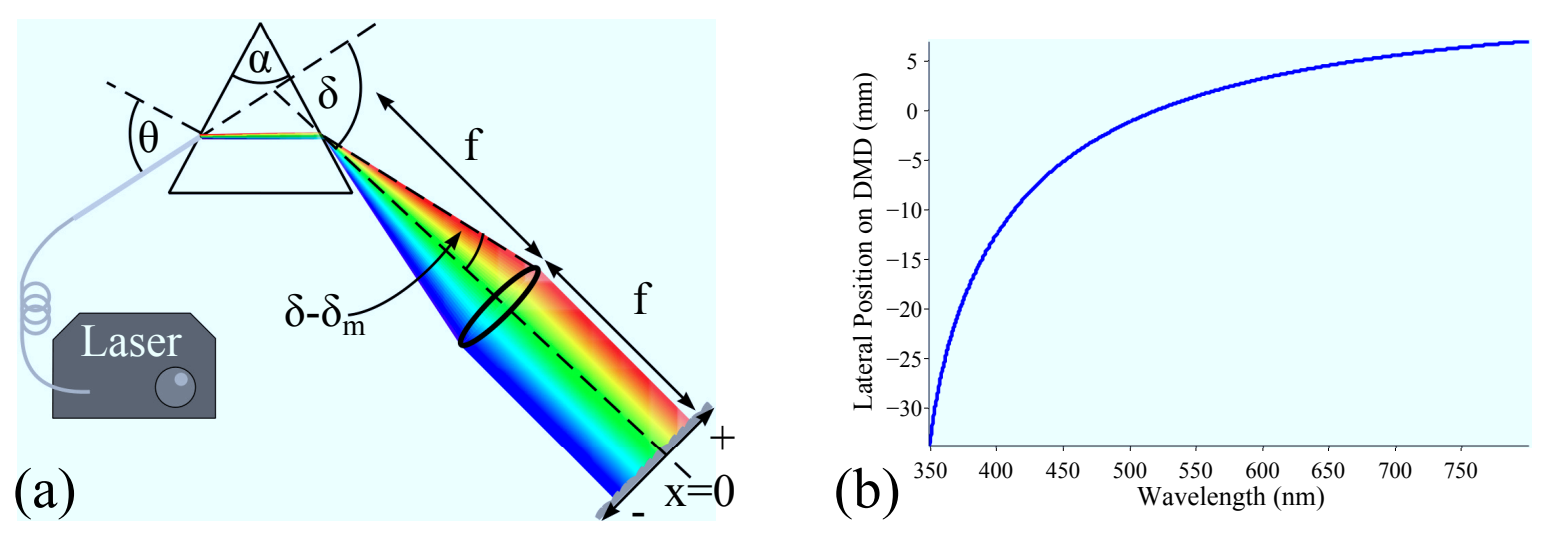

Figure 6. (a) Simplified optical system and (b) resulting lateral location on DMD.

The position on the DMD of a series of wavelengths, chosen to match the resolution of the spectrometer used in our experiments (Ocean Optics HR4000), was then calculated with equation 8 . These positions were converted to pixel column numbers, and the minimum and maximum wavelength in each individual column recorded. The resulting offset, of up to $\pm 7 \mathrm{~mm}$ is of the same order of magnitude as the size of a diffraction order and so contributes to the structure in the far-field.

The simulated and measured pattern for two wavelengths, at 482 and $617 \mathrm{~nm}$ and screen distance of $45 \mathrm{~cm}$ is shown in figure 7 . Note that the axes do not begin at zero due to the angular deviation caused by the mirror blaze. The diffraction pattern from the experimental system was observed in the laboratory by using the above system with all elements after the front face of the TIR prism removed. Columns of mirrors equivalent to 482 and $617 \mathrm{~nm}$ were selected and the pattern photographed on a paper screen placed $45 \mathrm{~cm}$ away from the DMD. This had a $5 \mathrm{~mm}$ grid printed on it for scale and is shown in figure 7.

The red stripe labelled ' $A$ ' in figure 7 is a surface reflection from the dispersing prism that could not be blocked without obscuring much of the diffraction pattern and should be ignored. In both figure $7(\mathrm{a})$ and (b) diffraction orders can be observed in both the horizontal $(x)$ and vertical $(y)$ directions. However, the vertical orders are much narrower than the horizontal. This is because each wavelength is focussed in the $x$-direction, leading to wide diffraction, but is not focussed in the $y$-direction.

At $+14 \mathrm{~cm}$ in figure 7 (a), and at label ' $\mathrm{B}$ ' in figure 7 the red and blue diffraction orders in the vertical direction are almost coincident. The adjacent vertical order, labelled ' $\mathrm{C}$ ', is also the brightest, but red and blue are not coincident. In addition, in the horizontal direction it can be clearly seen in figure 7 (a) that the power is spread equally over two adjacent spots, implying that these wavelengths are far from blazed.

In order to compare the vertical order spacing a vertical line-scan was taken through the red component of each image to isolate the contribution from the $617 \mathrm{~nm}$ pattern, and the peak locations found. The scale for the measured image was calibrated using the grid squares. This gave a mean peak spacing, across four orders, of $20.3 \mathrm{~mm}$ in both 


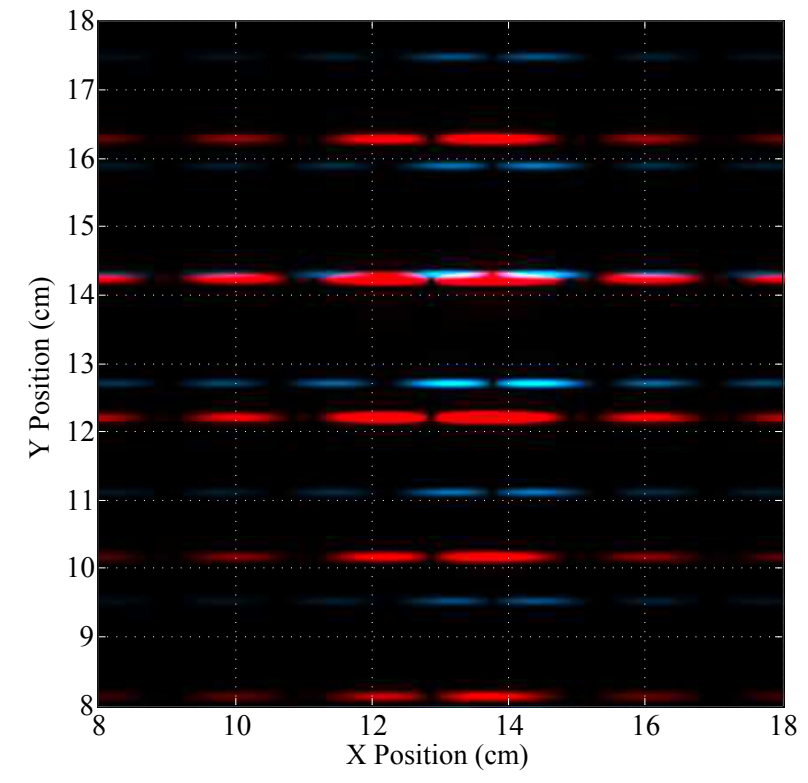

(a)

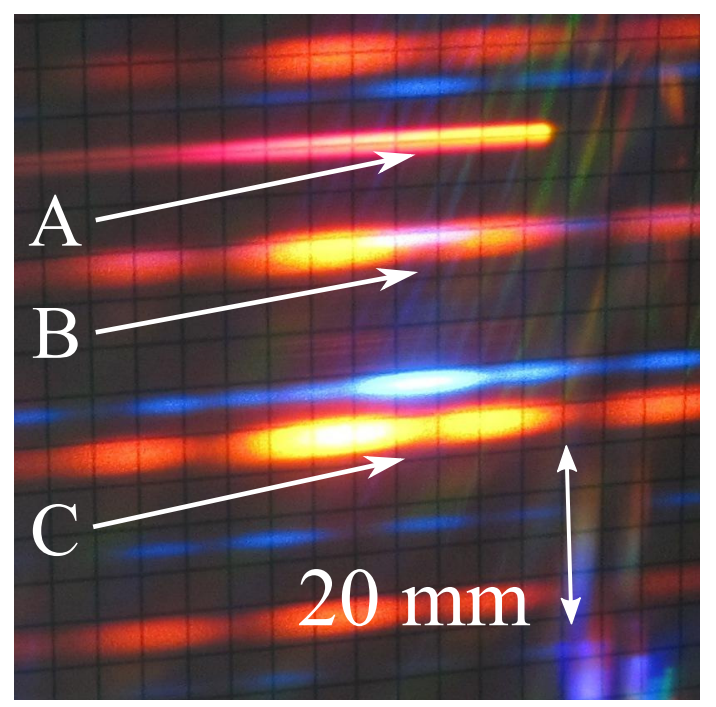

(b)

Figure 7. (a) Simulated and (b) recorded images at red and blue wavelengths. A Reflection from first prism. B - Coincident vertical order. C - Central, non-coincident order.

measurement $( \pm 0.1 \mathrm{~mm})$ and simulation.

Finally figure 8 shows a close-up of the bright region in the simulated diffraction pattern for for 200 equally spaced wavelengths between 450 and $650 \mathrm{~nm}$, and also the observed pattern in the laboratory. The simulated image was gamma corrected with a value of 0.7 to increase the brightness of dark areas relative to the central peak.

The full simulation shows that the majority of the power is still directed into the 


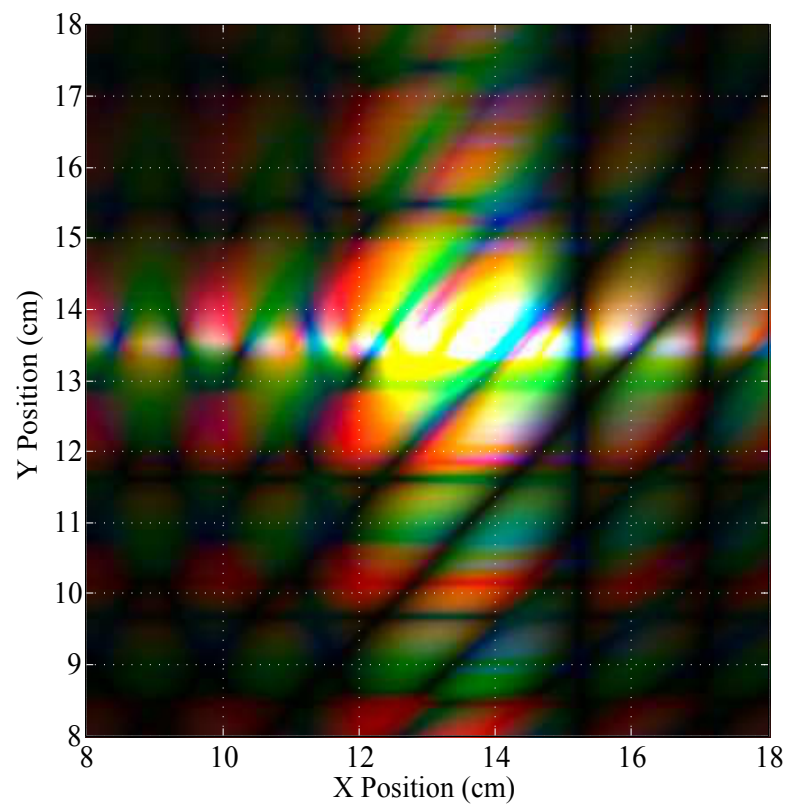

(a)

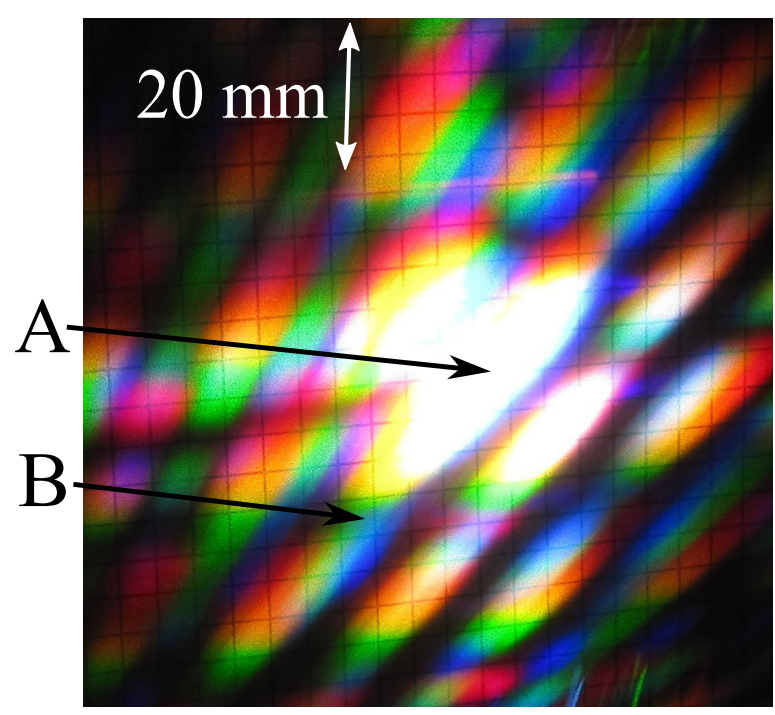

(b)

Figure 8. (a) Simulated and (b) recorded patterns for the entire available spectrum. A - Central order containing all wavelengths. B - secondary order containing a distinct band of wavelengths.

horizontal and vertical directions. However each individual order now appears smeared out in the diagonal direction due to two competing wavelength dependencies. The dominant effect is that within each diffraction order red wavelengths are spread further than blue in the far-field. This leads to the main strong diagonal shape of each individual order. 
The spatial wavelength spread caused by the prism dispersion leads to stronger color separation in the horizontal than vertical which makes separate orders appear to overlap. In the centre the orders overlap strongly, leading to bright white spots (labelled 'A'), whereas further out the overlap is less leading to areas of more distinct color (labelled ' $\mathrm{B}$ '). This is important for our application as the central region of the diffraction pattern contains significant power from all available wavelengths. A significant portion of this region can be captured and collimated by the second cylindrical lens and dispersion prism shown in figure 2 , and then focussed to a small spot by the cylindrical lens.

\subsection{Power}

Since the intended application this system is as an illumination source for biomedical applications we obtained a fibre and liquid light guide (Part numbers 495 ND and 495 FO, Karl Storz GmbH). These are typically used to deliver light into commercial medical instruments such as endoscopes or microscopes and have standardised light connectors. In addition these have larger apertures than a single fibre, reducing the difficulty of coupling sufficient light at all wavelengths. Both of the light guides were incorporated into the system and the output from each measured.

An important performance quantity for any illumination system is the output power and spectrum. For our system the supercontinuum determines the maximum available power and spectral envelope. Each subsequent component in the system has a transmission profile that will reduce the output power at each wavelength. To quantify these effects an integrating sphere and spectrometer (HR4000, Ocean Optics) were used to measure the spectrum of the raw supercontinuum and at points after significant components in the optical system. A more accurate power measurement was obtained using a broadband photodiode (M10, Coherent) in place of the integrating sphere and spectrometer. The spectral results are shown in figure 9 and the measured powers given in table 1 .

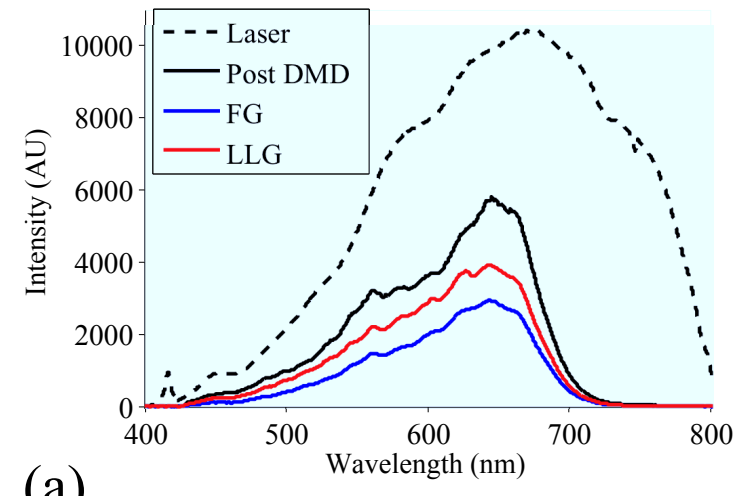

(a)

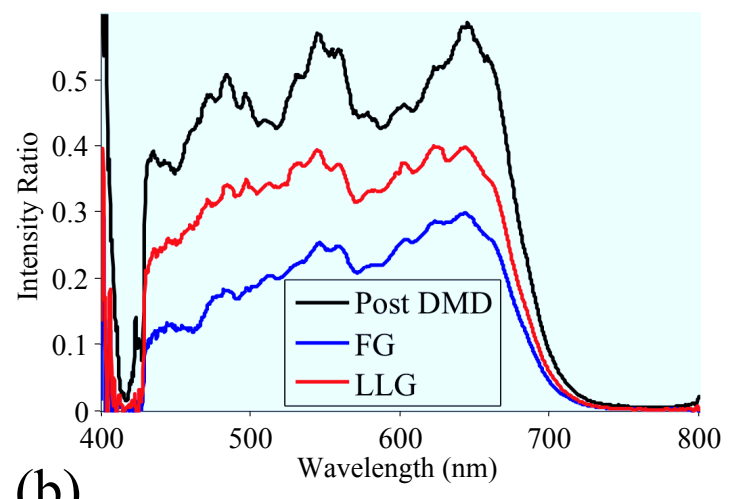

(b)

Figure 9. The raw supercontinuum spectrum and that measured at various points in the system. FG - Fibre light Guide. LLG - Liquid Light Guide. 


\begin{tabular}{cc}
\hline Component & Power $(\mathrm{W})$ \\
\hline Laser & 1.263 \\
Post DMD & 0.228 \\
Fibre Guide & 0.118 \\
Liquid Light Guide & 0.184 \\
\hline
\end{tabular}

Table 1. The total measured power in the complete spectrum when measured after various components

Although the supercontinuum generates power from $400 \mathrm{~nm}$, the power significantly increases above $500 \mathrm{~nm}$. Between 425 and $700 \mathrm{~nm}$, on a per-wavelength basis the transmission efficiency is approximately 50\% as shown in figure 9. The DMD package, including the windows, is only $68 \%$ efficient, so accounts for the majority of this loss. The remaining losses can be attributed to the high number (13) of air-glass surfaces that must be transited in the system. Above $700 \mathrm{~nm}$ there is a significant drop in power, likely because of the anti-reflection coatings on most of the components including the DMD window, which is coated to transmit light between 400 and $700 \mathrm{~nm}$. Both the fibre and liquid light guides were observed to have low throughput below $480 \mathrm{~nm}$. Supercontinuum systems based on mechanical slits instead of DMDs exhibit an approximately $30 \%$ loss in optical power $[3,4]$.

The power output of our system, at $184 \mathrm{~mW}$ is comparable to the quoted powers of commercial systems $(250 \mathrm{~mW}$ for the OneLight Spectra, $229 \mathrm{~mW}$ for the Optronic OL-490). We also compared the output to a standard $300 \mathrm{~W}$ Xenon arc lamp containing an infrared block filter, which after transmission through the fibre light guide produced $3.742 \mathrm{~W}$, which is approximately one order of magnitude larger than the supercontinuum system.

\subsection{Stability}

Supercontinuum generation relies on non-linear processes inside a photonic crystal fibre and these are susceptible to power fluctuations and changes in temperature. This can affect the power spectral density (PSD) of the output light and hence it was necessary to quantify the stability of the spectra our system could generate.

In particular we tested for major power fluctuations over timescales of typical acquisition periods for laparoscopic imaging systems [20], where individual exposure times are generally in the range of 0.1 to 1 second, and a complete acquisition across all bands could last up to 10 seconds. Hence the region of frequencies of interest spanned two orders of magnitude, from about $0.1 \mathrm{~Hz}$ to $10 \mathrm{~Hz}$.

We recorded two test spectra for a period of five minutes, sampling once every 50 ms. The two spectra are shown in figure 10; one consisted of the complete available spectrum while the other was four separate columns of pixels corresponding to peak wavelengths of 533,569, 615 and $663 \mathrm{~nm}$. Figure 10(b) shows the total intensity of 

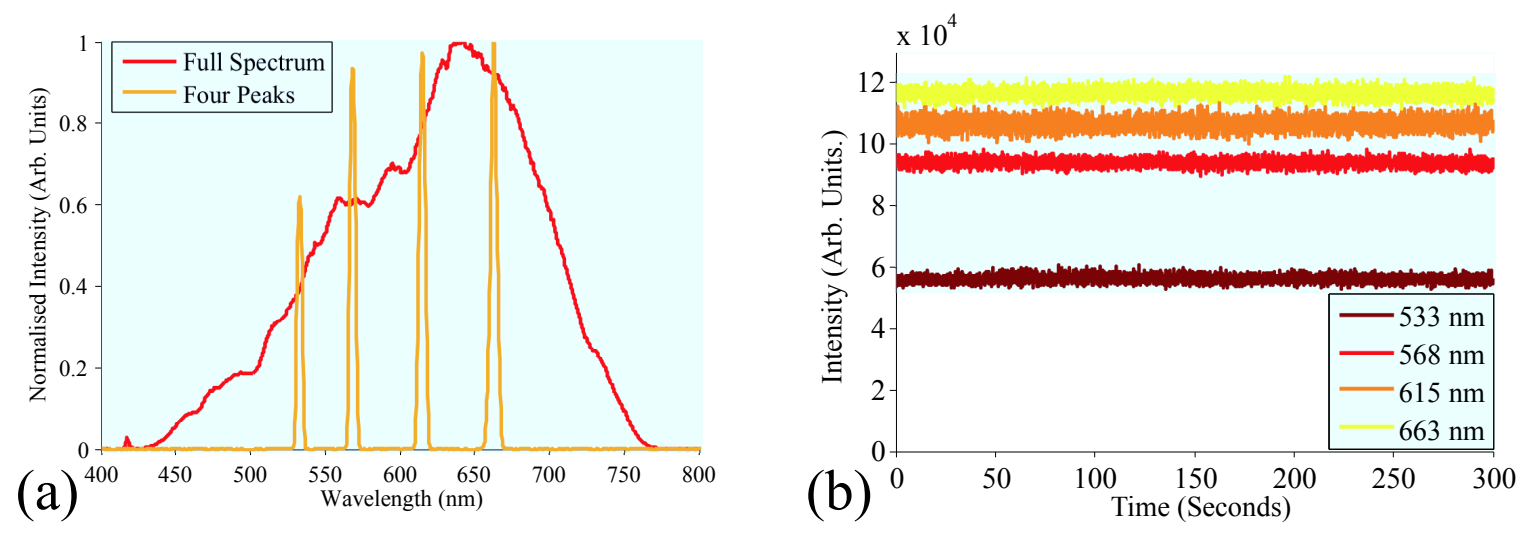

Figure 10. (a) Illustrative spectra used to record (b) the temporal intensity data used for measuring stability.
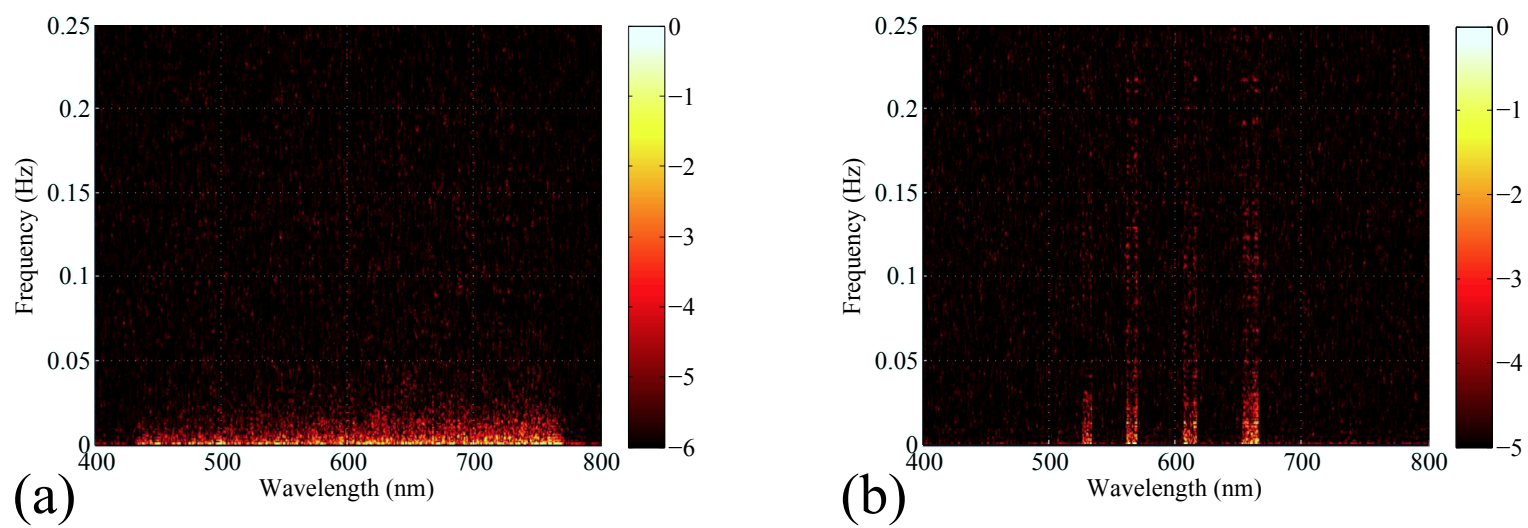

Figure 11. Wavelength domain spectrograms generated from the spectra in figure 10. The colour scale is presented on a $\log _{10}$ scale. The predominant red colour corresponds to a PSD of 0.005 .

these bands over the five minute period. There are no obvious fluctuations and the power appears to remain fairly constant. To ensure this was the case spectrograms were calculated by taking the autocovariance of the time series for each wavelength and then calculating the Fourier transform to obtain the PSD.

The resulting spectrograms are shown in figure 11. The PSD is shown on a logarithmic scale with a normalised maximum value of 1 . The only significant power shown in the image is near zero frequency. The frequency scale is only shown between 0 and $0.25 \mathrm{~Hz}$ because no significant power was observed at higher frequencies. From this we can conclude that the supercontinuum is fairly stable, with no long term drift or fluctuation apparent. On shorter timescales intensity variations may arise from the mechanisms used to produce the supercontinuum. 

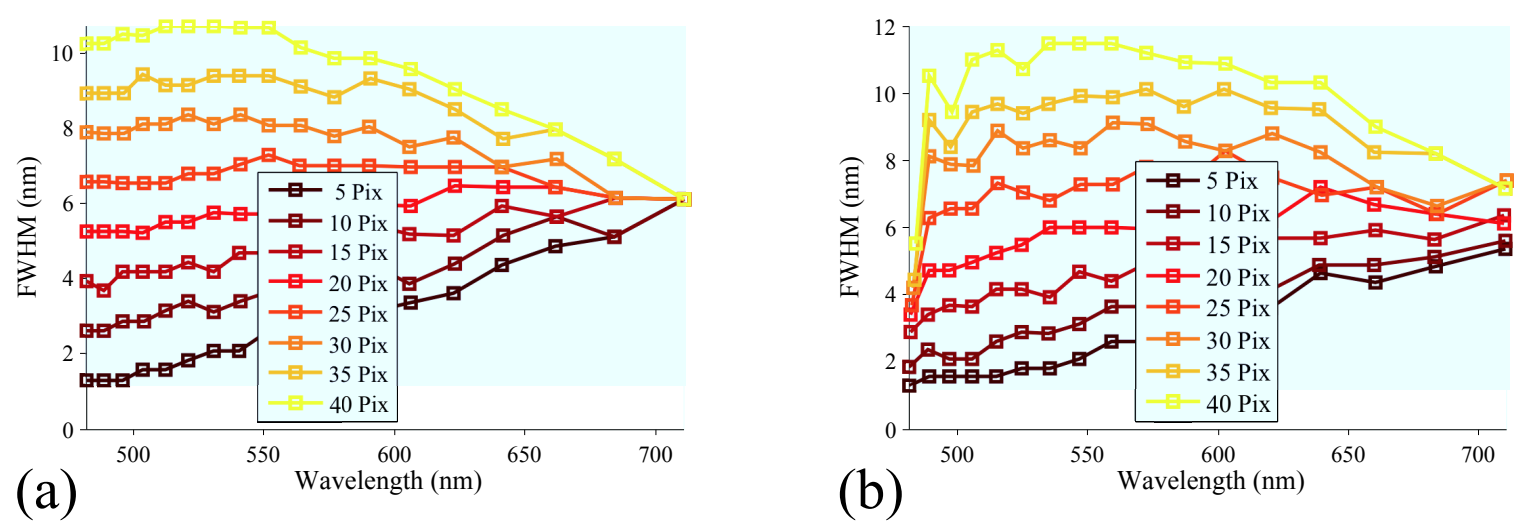

Figure 12. (a) Simulated and (b) measured bandwidths for a final column width of 5 pixels and starting widths shown in the legend $\left(q_{w}=q_{c}=1\right)$.

\subsection{Linearity and Resolution}

The non-linear dispersion of the prism results in an uneven distribution of wavelengths across the face of the DMD. Consequently, if a constant bandwidth or spacing is desired for imaging, then a non-constant column width and position must be used on the face of the DMD.

Using the Sellmeier equation analysis above a look-up table can be used to simulate the response of the system. The spectral bandwidth of a contiguous group of pixel columns is simply the minimum wavelength falling on the leftmost column subtracted from the maximum wavelength in the rightmost column. To linearise the bandwidth and spacing the column addresses would have to take the form of the inverse of equation 8 , but as noted above this cannot be inverted. Instead a scaled power law was implemented so that an appropriate power could be used to linearise the system as much as possible. This algorithm requires seven parameters; the start and stop column of the DMD, $c_{1}$ and $c_{2}$, are chosen which correspond to the desired overall wavelength range, along with $n$, the number of desired bands, and a start and stop column width $w_{1}$ and $w_{2}$. The central column and width of band $i$ can then be found from

$$
w_{i}=\left\lceil w_{1}+\left(\frac{i}{n}\right)^{q_{w}}\left(w_{2}-w_{1}\right)\right\rceil \quad c_{i}=\left\lceil c_{1}+\left(\frac{i}{n}\right)^{q_{c}}\left(c_{2}-c_{1}\right)\right\rceil
$$

where $q_{w}$ and $q_{c}$ are the independent factors that tune the bandwidth and spacing respectively. The left- and rightmost column addresses for each band can then be found from $c_{i} \pm w_{i} / 2$. Figure 12 shows both the simulated and measured results if both $q_{w}$ and $q_{c}$ are equal to unity, giving linearly spaced columns on the DMD, but with different starting column widths. If a constant width of 5 pixels (bottom lines in figure 12) is used, then the bandwidth approximately increases by a factor of 5 over the full wavelength range. The band spacing is also highly non-linear, as can be seen by the closer spacing of data points towards lower wavelengths.

In order to improve the band spacing a range of possible $q_{c}$ values was investigated. Intuitively, columns should be spaced closer together at longer wavelengths as these are 


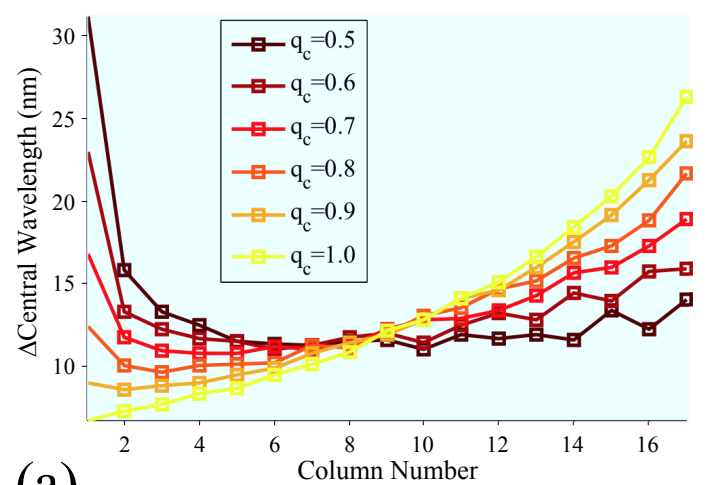

(a)

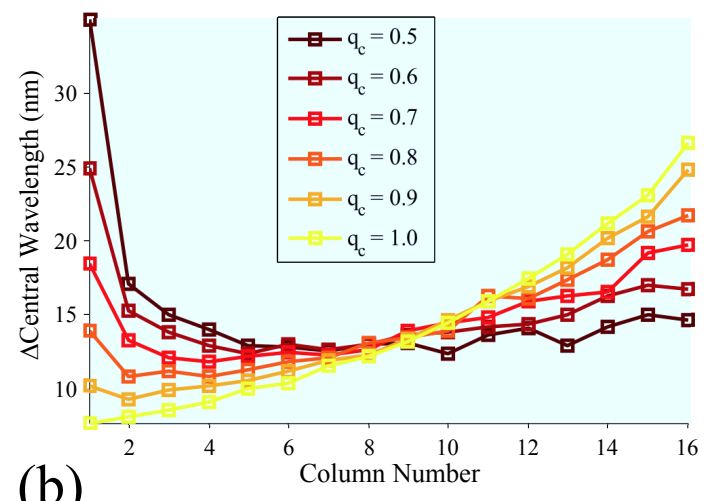

(b)

Figure 13. (a) Simulated and (b) measured band spacing for various values of $q_{c}$ shown in the legend and a constant column width of 5 pixels.

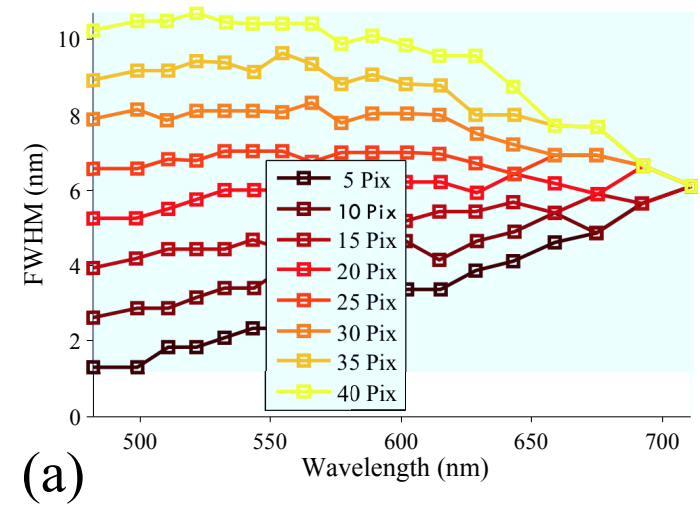

(a)

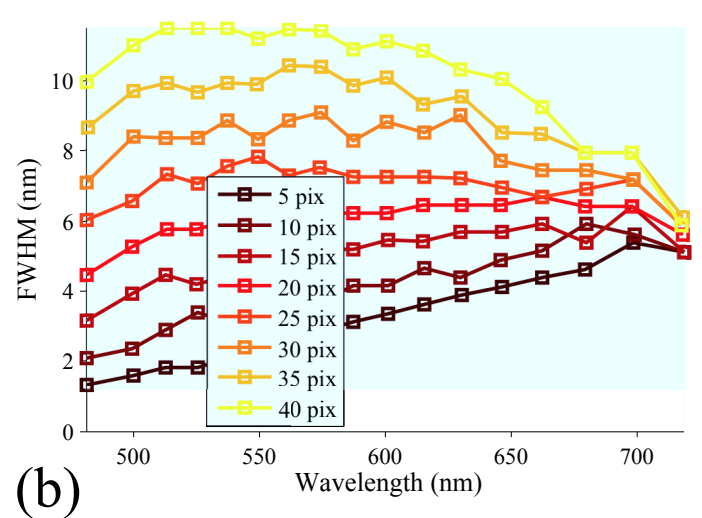

Figure 14. (a) Simulated and (b) measured FWHM for the system with $q_{w}=q_{c}=0.7$. The evenly spaced data points indicate roughly constant band-spacings.

more closely spaced on the DMD. As $(c 2-c 1) e<0$ in equation 11 , this implies that $q_{c}$ should be less than one. Hence a range of values from 0.5 to 1.0 was chosen, and the results are shown in figure 13 . A value of $q_{c}=0.7$ produces the flattest overall spacing. If we then use the same value for $q_{w}$ and redraw figure 12, we can see that a start to end column-width ratio of five still produces the flattest bandwidth, but now also has a roughly constant band spacing. This is shown in figure 14 .

The bandwidth curves are not smooth, even when simulated. This is an artefact of the integer column widths, given in equation 11. As illustrated in figure 15 the bandwidth for constant column widths increases smoothly. In order to produce a constant spectral bandwidth the stop width must be less than the start width. Each time the column width decreases by one, the bandwidth jumps to the neighbouring curve in figure 15, producing the discontinuities in the other bandwidth plots. This is shown by the solid black line, which is for a starting width of four pixels and a stop width of one pixel. The line overlaps each other curve on the graph for a small region of wavelengths, before jumping to the next one. 


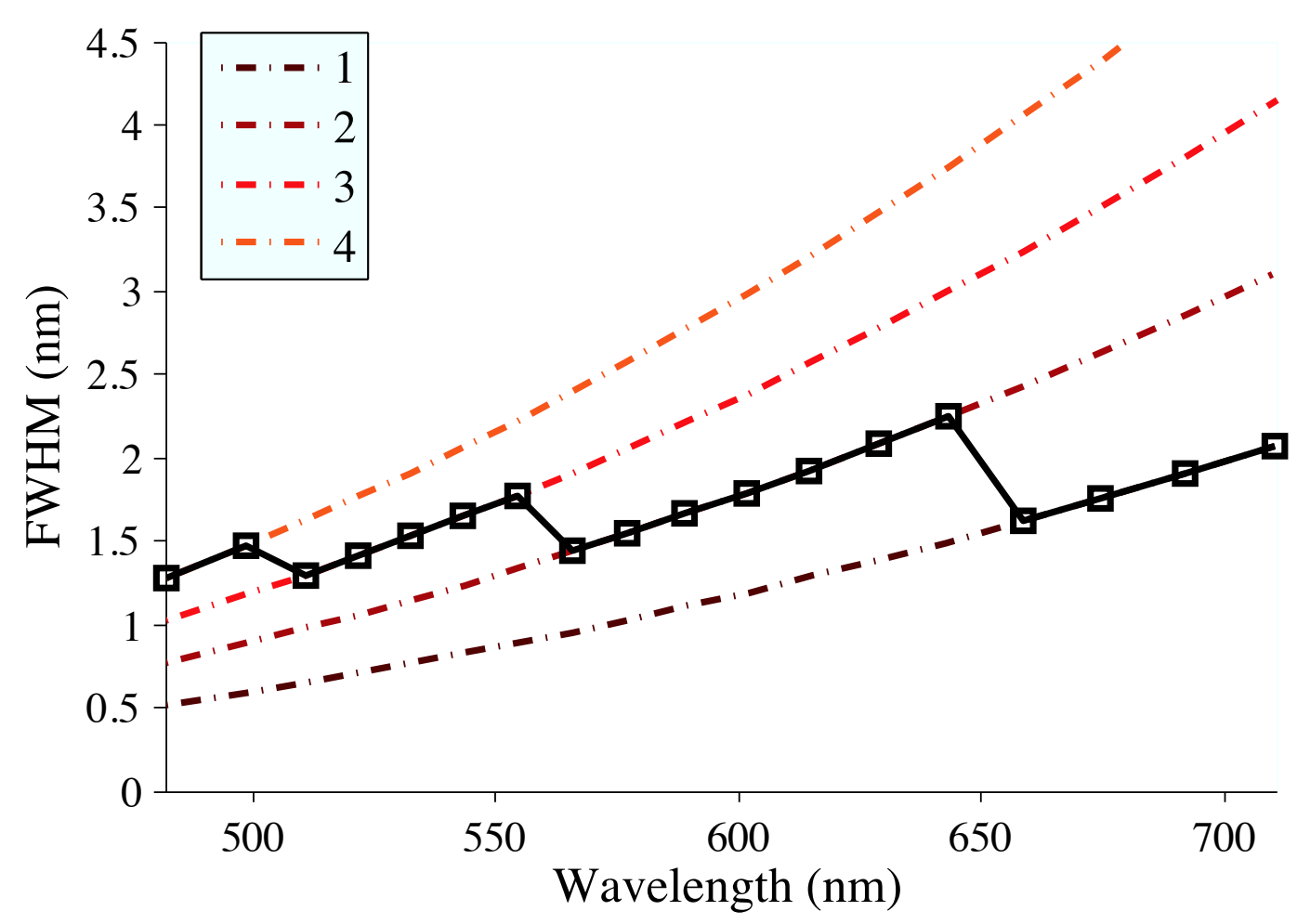

Figure 15. Spectral bandwidth plotted for constant column widths of 1 - 4 pixels (dotted lines) and a variable width that starts at 4 pixels and steps down to 1 pixel (Black solid line).

To illustrate the results of this linearization process the actual measured spectra used to generate figures 12 and 14 for a starting width of 25 pixels are shown in figure 16 . This shows that the system can be tuned to produce approximately evenly spaced illumination bands of a roughly constant width of $6 \mathrm{~nm}$. This compares well with the resolutions of commercial systems (10 $\mathrm{nm}$ for the OneLight Spectra and $5 \mathrm{~nm}$ for the Optronic OL-490), and can be reduced further if longer wavelengths are not required.

\section{Conclusions}

We have constructed a dynamic hyperspectral illumination system using a DMD to spectrally filter a supercontinuum laser source. The incorporation of a TIR prism into the system significantly reduces the complexity of the optics required to collect light from the DMD. The resulting system prototype displays similar output power and spectral resolution to existing commercial systems. We have also fully analysed the diffraction pattern of the DMD, modelling it as a two-dimensional blazed grating instead of approximating it in one-dimension. Finally we have discussed how the non-linear dispersion prism affects the system response and demonstrated a possible correction scheme which allows different spectral bands to be selected. The generation 

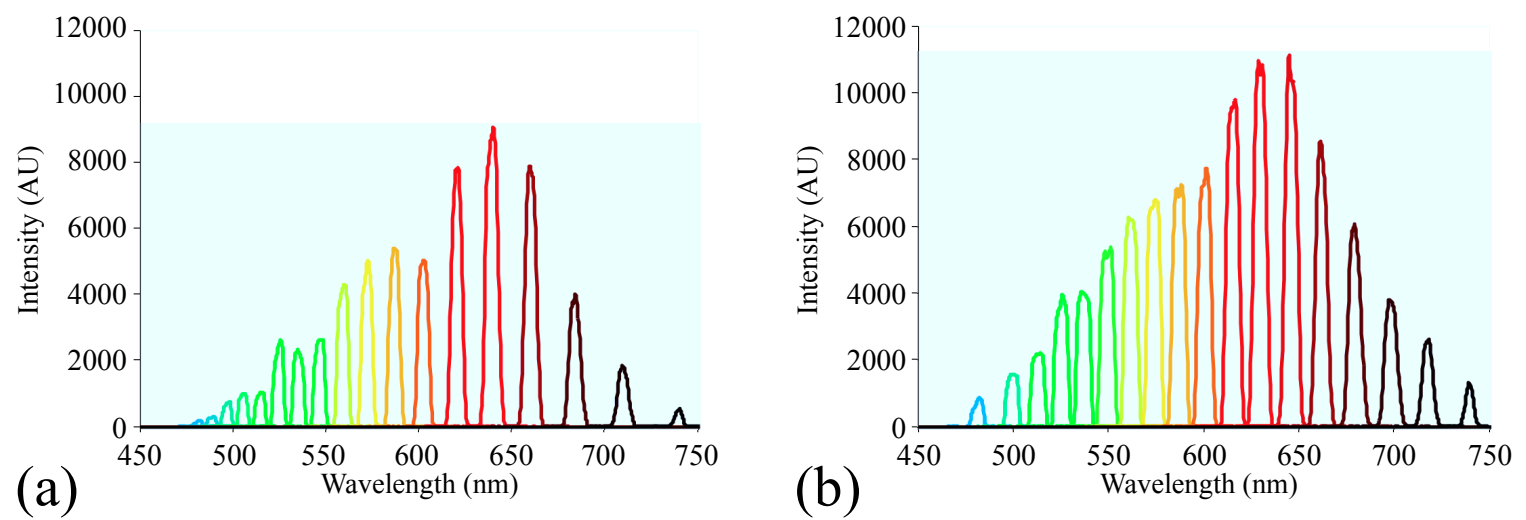

Figure 16. Spectra generated for starting and stop column widths of 20 and 5 pixels, colored by peak wavelength, (a) without correction and (b) after linearisation.

of such custom illumination spectra will enable the realisation of multispectral imaging modalities in microscopy and minimally invasive surgery.

\section{Acknowledgements}

We gratefully acknowledge support from the UK EPSRC (grant EP/E06342X/1), and Ocean Thin Films for the TIR prism.

\section{References}

[1] Michael F. Becker, Jinyang Liang, Rudolph N. Kohn, and Daniel J. Heinzen. High-precision laser beam shaping using binary-amplitude DLP spatial light modulators. In Emerging Digital Micromirror Device Based Systems and Applications II, volume 7596. SPIE, 2010.

[2] Jason Geng. DLP-based structured light 3d imaging technologies and applications. In Emerging Digital Micromirror Device Based Systems and Applications III, volume 7932. SPIE, 2011.

[3] C. Dunsby, P. M. P. Lanigan, J. McGinty, D. S. Elson, J. Requejo-Isidro, I. Munro, N. Galletly, F. McCann, B. Treanor, B. Onfelt, D. M. Davis, M. A. A. Neil, and P. M. W. French. An electronically tunable ultrafast laser source applied to fluorescence imaging and fluorescence lifetime imaging microscopy. Journal of Physics D: Applied Physics, 37(23):3296-3303, 2004.

[4] P. M. P. Lanigan, C. Dunsby, J. McGinty, D. S. Elson, J. Requejo-lsidro, I. Munro, N. Galletly, B. Treanor, B. Onfelt, F. McCann, D. M. Davis, M. A. A. Neil, and P. M. W. French. An electronically tunable ultrafast laser source applied to fluorescence imaging and fluorescence lifetime imaging microscopy. In Conference on Lasers and Electro-Optics, volume 2, pages $1366-1368,2005$.

[5] Nicholas MacKinnon, Ulrich Stange, Pierre Lane, Calum MacAulay, and Mathieu Quatrevalet. Spectrally programmable light engine for in vitro or in vivo molecular imaging and spectroscopy. Appl. Opt., 44(11):2033-2040, 42005.

[6] Michael L. Mangum, Michel Saint-Cyr, Eleanor F. Wehner, Abhas Thapa, Edward Livingston, and Karel J. Zuzak. Visible to NIR DLP hyperspectral imaging system for surgical utility using inherent chromophores and fluorescent probes. In Emerging Digital Micromirror Device Based Systems and Applications III, volume 7932. SPIE, 2011.

[7] Eleanor Wehner, Abhas Thapa, Edward Livingston, and Karel Zuzak. Nir dlp® hyperspectral 
imaging system for medical applications. In Emerging Digital Micromirror Device Based Systems and Applications III, volume 7932. SPIE, 2011.

[8] G. McConnell, S. Poland, and J. M. Girkin. Fast wavelength multiplexing of a white-light supercontinuum using a digital micromirror device for improved three-dimensional fluorescence microscopy. Review of Scientific Instruments, 77(1):013702, 2006.

[9] Joseph P Rice, Jorge E Neira, Michael Kehoe, and Rand Swanson. Dmd diffraction measurements to support design of projectors for test and evaluation of multispectral and hyperspectral imaging sensors. In Emerging Digital Micromirror Device Based Systems and Applications, volume 7210. SPIE, 2009.

[10] John M. Dudley, Goery Genty, and Stephane Coen. Supercontinuum generation in photonic crystal fiber. Reviews of Modern Physics, 78(4):1135-50, 2006.

[11] Larry J Hornbeck. Active yoke hidden hinge digital micromirror device. Patent 5,535,047, Texas Instruments, 71996.

[12] Nadim Maluf and Kirt Williams. An Introduction to Microelectromechanical Systems Engineering. Artech House, 2nd edition, 2004.

[13] C. H. Chuang and Y. L. Lo. Digital programmable light spectrum synthesis system using a digital micromirror device. Appl. Opt., 45(32):8308-8314, 2006.

[14] S. W. Brown, J. P. Rice, J. E. Neira, B. C. Johnson, and J. D. Jackson. Spectrally tunable sources for advanced radiometric applications. Journal of Research of the National Institute of Standards and Technology, 111(5):401-410, 92006.

[15] Joseph P Rice and David W Allen. Hyperspectral image compressive projection algorithms. In Emerging Digital Micromirror Device Based Systems and Applications, volume 7334. SPIE, 2009.

[16] D Allen and Joseph P Rice. Hyperspectral projection of a coral reef scene using the nist hyperspectral image projector. In Algorithms and Technologies for Multispectral, Hyperspectral, and Ultraspectral Imagery XV, volume 7334. SPIE, 2009.

[17] M. C. Hutley. Diffraction gratings. Techniques of Physics. Academic Press, 1982.

[18] Texas Instruments. Using lasers with dlp dmd technology. TI DN 2509927, Texas Instruments, 9 2008.

[19] Eugene Hecht. Optics. Addison Wesley, 3rd edition, 1998.

[20] Kevin R Koh, Tobias C Wood, Robert D Goldin, Guang-Zhong Yang, and Daniel S Elson. Visible and near infrared autofluorescence and hyperspectral imaging spectroscopy for the investigation of colorectal lesions and detection of exogenous fluorophores. In Advanced Biomedical and Clinical Diagnostic Systems VII, 2009. 